\title{
Microwave wireless power transmission technology index system and test evaluation methods
}

\author{
Changfu Wang ${ }^{1}$, Wanli Xu' ${ }^{1}$, Chunlin Zhang ${ }^{2}$, Mengyi Wang ${ }^{1}$ and Xudong Wang ${ }^{\text {* }}$
}

\author{
${ }^{*}$ Correspondence: \\ energywxd@163.com \\ ${ }^{1}$ Institute of System \\ Engineering, Academy \\ of Military Science, Beijing, \\ China \\ Full list of author information \\ is available at the end of the \\ article
}

\begin{abstract}
Microwave wireless power transmission technology has broad application prospects in improving the endurance and range of unmanned equipment. It is also of great significance in the field of the Internet of things, which can effectively solve the problem of energy supply for devices in the Internet of things. In addition, using vehicle-mounted microwave wireless power transmission to power multi-objective unmanned aerial vehicle swarms and to build an air-ground integrated power transmission network. In this study, the corresponding technical index system and test evaluation methods, which are conducive to promoting the development and progress of this technology, were established. The topology of a microwave wireless power transmission system was analyzed, and technical evaluation indices were developed for microwave wireless power transmission systems, signal generators, power amplifiers, transceiver antennas, and rectifier circuits. Test evaluation methods were established consisting of test equipment, methods, and processes to provide powerful technical means for the development of microwave wireless power transmission systems.
\end{abstract}

Keywords: Wireless power transmission, Microwave, Technical index, Test methods

\section{Introduction}

\subsection{Background and significance}

Microwave wireless power transmission is a technology that uses microwave devices to convert electrical energy into electromagnetic energy and wirelessly transmit microwave electromagnetic energy in space through a transmitting antenna, and converts electromagnetic energy into electric energy and supplies it to the electric load after rectification, filtering, and other transformations [1,2]. Microwave wireless power transmission technology has the advantages of high transmission power, strong environmental adaptability, and flexible deployment of focus and scattering [3], making it suitable for powering unmanned equipment over long distances in complex environments to improve the endurance and range of such equipment $[4,5]$. Microwave wireless power transmission is also of great significance in the field of the Internet of things [6, 7], which can effectively solve the problem of energy supply for devices in the Internet of things [8, 9]. As shown in Fig. 1, a vehicle-mounted microwave wireless power transmission system author(s) and the source, provide a link to the Creative Commons licence, and indicate if changes were made. The images or other third party material in this article are included in the article's Creative Commons licence, unless indicated otherwise in a credit line to the material. If material is not included in the article's Creative Commons licence and your intended use is not permitted by statutory regulation or exceeds the permitted use, you will need to obtain permission directly from the copyright holder. To view a copy of this licence, visit http:// creativecommons.org/licenses/by/4.0/. 


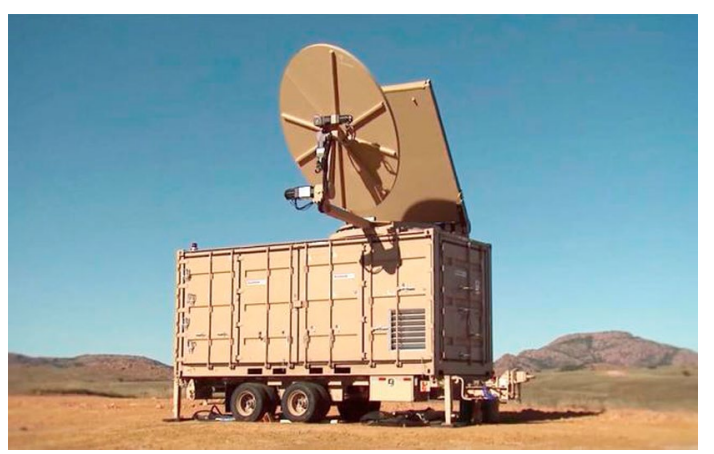

Fig. 1 Vehicle-mounted microwave wireless power transmission system

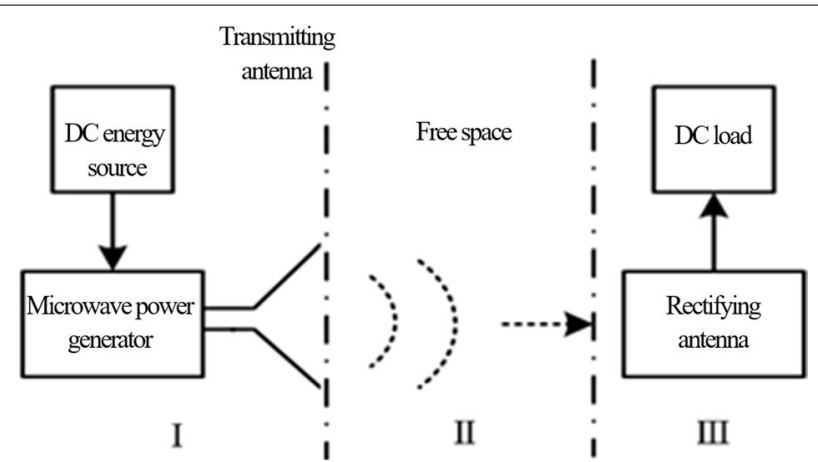

Fig. 2 Diagram of microwave wireless power transmission system topology

can build an air-ground integrated electrical power transmission network to guarantee an accompanying power supply for unmanned equipment. However, most current microwave wireless power transmission systems have a short transmission distance, low transmission power level, and a low degree of intelligence and miniaturization [4]. Meanwhile, the large size of the system and devices and the lower transmission efficiency and power limit the further development and application of microwave wireless power transmission technology [10]. In this study, the focus was on the technical index system and test evaluation methods of microwave wireless power transmission to provide powerful technical means for the development of microwave wireless power transmission systems.

\subsection{Microwave wireless power transmission system topology}

The basic topology of the microwave wireless power transmission system is shown schematically in Fig. 2, which depicts the DC energy source, microwave power generator (microwave signal generator and power amplifier), transmitting antenna, free space, rectifying antenna (receiving antenna and rectifying circuit), and DC load [11, 12].

The DC energy source provides energy for the microwave power transmission system. The signal generator is used to generate low-power microwave signals, and the power amplifier amplifies the generated small signals to output high-power microwave signals. The transmitting antenna converts the fed high-power microwave energy into 
electromagnetic waves that radiate into free space. The receiving antenna converts the electromagnetic wave of the corresponding frequency band in the area into a microwave signal in the circuit and sends it to the rectifier circuit. The receiving antenna and rectifier circuit are often combined into a rectifier antenna. The microwave rectifier circuit uses the nonlinear effect of the rectifier diode to convert the received radiofrequency energy into direct current energy (RF-DC) to power the load.

\section{Methods}

\subsection{Signal generator}

\subsubsection{Technical index}

The technical index of the signal generator mainly includes the operating frequency and efficiency. The operating frequency is the nominal frequency value of the frequency source under the given operating conditions and is also called the "carrier frequency." The efficiency of a signal generator represents its ability to convert an input DC signal into a microwave signal [13]. In terms of mathematical and physical laws, the transmission process of wireless energy is closely related to the frequency of electromagnetic waves - the higher the frequency of the electromagnetic waves, the better their directivity and the less the spatial attenuation. The frequencies commonly used for energy transmission in the microwave band are $2.45,5.8$, and $35 \mathrm{GHz}$ or higher. Therefore, it is critical to evaluate whether the signal generated by the generator meets the microwave power transmission requirements.

\subsubsection{Test and evaluation methods}

The main equipment used for the performance test of the signal generator comprised a DC energy source, spectrum analyzer, and DC power meter. The spectrum analyzer is the basic tool for observing the signal. It can locate and measure the frequency of the signal and display it graphically. The test diagram is shown in Fig. 3.

The steps involved are as follows.

Step 1 Connect the signal generator being tested to the circuit, as shown in Fig. 3, and connect the spectrum analyzer to the signal generator through a coaxial cable.

Step 2 Turn on the DC power source and signal generator.

Step 3 Record the DC power meter readings $P_{\text {sin }}$.

Step 4 Use the marker function of the spectrum analyzer to find the maximum power point, which is the output power of the signal generator, and record the frequency $f_{s}$ corresponding to the point, which is the working frequency of the signal generator.

The efficiency of the signal generator is calculated using

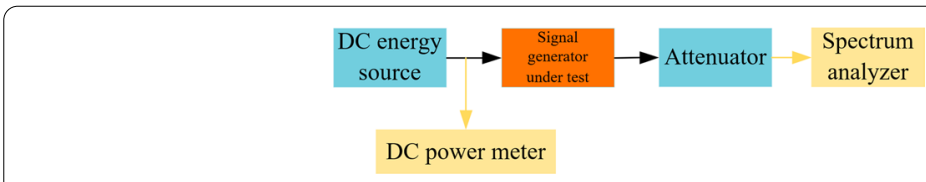

Fig. 3 Signal generator test chart 


$$
\eta_{s}=\frac{P_{\text {sin }}}{10 \lg \left(1000 \times P_{\text {sout }}\right)}
$$

where $\eta_{s}$ is the efficiency of the signal generator, $P_{\text {sin }}$ is the DC input power of the signal generator $(\mathrm{W})$, and $P_{\text {sout }}$ is the output power of the signal generator $(\mathrm{dBm})$.

\subsection{Power amplifier}

\subsubsection{Technical index}

The output power, power gain, and efficiency of the power amplifier directly affect the overall performance of the system. The main function of the power amplifier is to amplify small signals and then output high-power microwave signals. The power gain is the ratio of the power amplifier output power to the input power and is used to indicate the signal amplification capacity. A power amplifier is an important component of a microwave wireless power transmission system, and its efficiency affects the system transmission efficiency directly.

\subsubsection{Test and evaluation methods}

Power amplifier performance testing uses equipment comprising a microwave signal source, isolator, directional coupler, power meter, attenuator, and spectrum analyzer. Referring to GJB 8125-2013, ignoring circuit losses, the test diagram of the power amplifier is shown in Fig. 4.

The steps involved in testing are as follows.

Step 1 Connect the instrument to the amplifier being tested using a coaxial connector and measurement cable, as shown in Fig. 4.

Step 2 Adjust the frequency of the microwave signal source to a specified value and apply a specified bias to the amplifier being tested.

Step 3 Change the output power of the microwave signal source so that the input power to the amplifier reaches the specified value.

Step 4 Read and record the reading $P_{\mathrm{ai}}$ of microwave power meter 1 , the reading $P_{\mathrm{ao}}$ of microwave power meter 2 , and the reading $P_{\text {ad }}$ of the DC power meter.

The power gain of the power amplifier is calculated as follows.

$$
G_{p}=P_{\mathrm{ao}}-P_{\mathrm{ai}}
$$

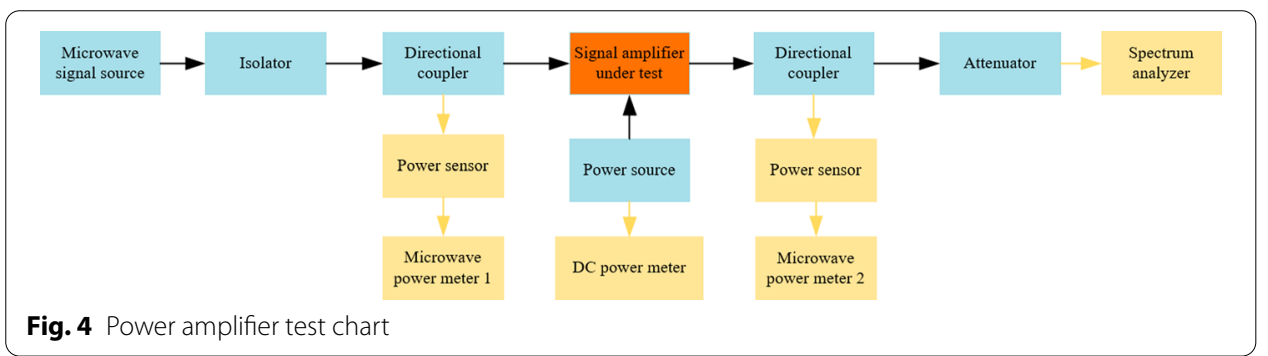


where $G_{p}$ is the gain of the power amplifier $(\mathrm{dB}), P_{\mathrm{ao}}$ is the microwave signal power amplified by the power amplifier $(\mathrm{dBm})$, and $P_{\mathrm{ai}}$ is the microwave signal power before amplification $(\mathrm{dBm})$.

The coefficient of the directional coupler is $C$, and the efficiency of the power amplifier is calculated as

$$
\eta_{p}=\frac{C \times P_{\mathrm{ao}}}{10 \lg \left(1000 \times P_{\mathrm{ad}}\right)} \times 100 \%
$$

where $\eta_{p}$ is the efficiency of the power amplifier, and $P_{\text {ad }}$ is the input power of the power amplifier DC power supply (W).

\subsection{Transceiver antenna}

\subsubsection{Technical index}

Because the wireless link from the transmitting antenna to the receiving antenna is an uncontrollable open loop, the performance depends entirely on the transmitting and receiving antenna performance and mutual matching. The technical indices of the transmitting and receiving antennas are mainly impedance characteristics and directional characteristics. The impedance characteristics include operating frequency and efficiency. Directional characteristics include directional map and gain [14]. In the microwave wireless power transmission system for moving targets, the transmitting antenna must change the transmitting direction of the beam according to the motion state of the target to realize the control of the beam direction and beam power [15]. If the energyreceiving equipment is a moving target with limited weight-bearing capacity, such as an unmanned aircraft [16], the lightness of the receiving end also must be considered. Therefore, the operating range and power density of the transmitting antenna are among the main technical indicators for evaluating performance.

The efficiency of the transmitting and receiving antenna is the ratio of the radiated power to the active power input to the antenna, which is closely related to the directional characteristics. In addition, because of electromagnetic wave propagation losses in free space [17], the transmitting and receiving antenna efficiency of a microwave wireless energy transmission system is defined as the ratio of the RF energy received by the receiving antenna to the energy input to the transmitting antenna. The operating range of the transmitting antenna refers to the antenna azimuth and pitch angle range. The power density is defined as the ratio of the power received by the receiving antenna to its mass and area.

\subsubsection{Test and evaluation methods}

The equipment required for transceiver antenna performance testing include a microwave signal source, antenna turn table controller, spectrum analyzer, microwave power meter, power sensor, and shaft angle encoder. The layout of the transmitting and receiving antenna performance test is shown in Fig. 5. Power meters 1 and 2 measure the RF power input to the transmitting antenna and the RF power received by the receiving antenna, respectively. The horizontal and vertical effective radiation ranges of the transmitting antenna were measured using the shaft angle encoder.

The steps involved are as follows. 


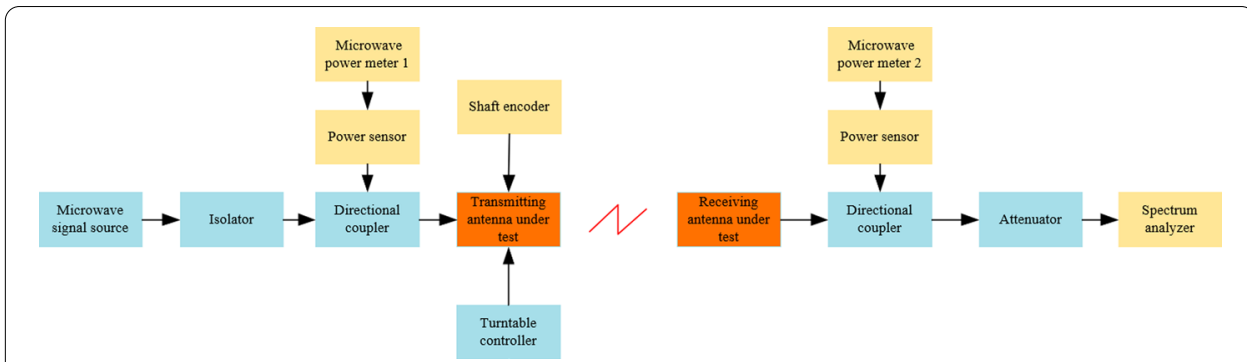

Fig. 5 Transceiver antenna test chart

Step 1 Connect the transmitting and receiving antennas being tested to the test circuit in accordance with Fig. 5.

Step 2 Set the microwave signal source frequency to the specified value.

Step 3 Read the spectrum analyzer frequency, which is the operating frequency $f$ of the transmitting and receiving antennas, and set power meters 1 and 2 to measure the signal frequency to $f$.

Step 4 Turn the turntable controller horizontally and record the shaft angle encoder readings as well as the power meter 1 and 2 readings.

Step 5 Repeat step 4 until the power meter 2 reading is zero or the rotation angle is $180^{\circ}$.

Step 6 Turn the rotary table controller vertically and record the shaft angle encoder reading as well as the power meter 1 and 2 readings.

Step 7 Repeat step 6 until the reading of power meter 2 is zero or the rotation angle is $180^{\circ}$.

The efficiency of the transceiver antenna is calculated as

$$
\eta_{\mathrm{tr}}=\left(P_{\mathrm{tr}}-P_{\mathrm{tt}}\right) \times 100 \%
$$

where $\eta_{\mathrm{tr}}$ is the transmitting and receiving antenna efficiency, $P_{\mathrm{tr}}$ is the measured input RF power of the transmitting antenna $(\mathrm{dBm})$, and $P_{\mathrm{tt}}$ is the measured output RF power of the receiving antenna $(\mathrm{dBm})$.

The mass power density of the receiving antenna is $\rho_{\mathrm{rm}}=\frac{C \times P_{\mathrm{tr}}}{m_{r}}(\mathrm{dBm} / \mathrm{kg})$, and the area power density is $\rho_{\mathrm{rs}}=\frac{C \times P_{\mathrm{tr}}}{s_{r}}\left(\mathrm{dBm} / \mathrm{m}^{2}\right)$.

The horizontal and vertical rotation angle and power meter 2 value change relationship curve (that is, the relationship between the received power and the transmitting antenna rotation angle) is plotted, and then the transmitting antenna orientation and pitch working range are determined. The maximum value of the shaft angle encoder recorded in step 5 is taken as $A_{\text {hmax }}$, and the minimum value as $A_{\mathrm{hmin}}$. Then, the horizontal working range of the transmitting antenna is $A_{\mathrm{h}}=A_{\mathrm{hmax}}-A_{\mathrm{hmin}}$. The maximum value of the shaft angle encoder recorded in step 7 is taken as $A_{\mathrm{vmax}}$ and the minimum value as $A_{\mathrm{vmin}}$. Then, the horizontal working range of the transmitting antenna is $A_{\mathrm{v}}=A_{\mathrm{vmax}}-A_{\mathrm{vmin}}$. 


\subsection{Rectifier circuit}

\subsubsection{Technical index}

The microwave rectifier circuit uses the nonlinear effect of the rectifier diode to convert the received microwave energy into DC energy to power the load. The core technical index for rectifier circuits is power conversion efficiency [18]. This efficiency is related to the characteristic parameters of the rectifier diode, DC load, and degree of impedance matching [19].

\subsubsection{Test and evaluation methods}

The equipment required for rectifier circuit performance testing comprise a microwave signal source, microwave power meter, load resistor, and DC power meter. The test layout is shown in Fig. 6.

The steps involved in the testing process are as follows.

Step 1 Connect the transmitting and receiving antennas being tested to the test circuit in accordance with Fig. 6.

Step 2 Set the microwave signal source frequency to a specified value.

Step 3 Read the frequency at the maximum power point of the spectrum analyzerthe frequency of the power meter measurement signal is set to that value.

Step 4 Record the value of the microwave power meter $P_{\mathrm{cr}}$, which is the RF power input to the rectifier circuit, and record the DC power meter reading $P_{\text {load }}$.

The efficiency of the rectifier circuit $\eta_{\text {re }}$ is calculated using

$$
\eta_{\mathrm{re}}=\frac{P_{\text {load }}}{C \times 10 \lg \left(1000 \times P_{\mathrm{cr}}\right)} \times 100 \%
$$

where $P_{\text {load }}$ is the rectified DC power $(\mathrm{W})$, and $P_{\mathrm{cr}}$ is the microwave power before rectification $(\mathrm{dBm})$.

\subsection{Microwave wireless energy transfer system}

\subsubsection{Technical index}

The technical indicators of the microwave wireless power transfer system are shown in Fig. 7, which include power class, efficiency class, and size class indicators [20, 21]. The power index includes the power of the main links of the system, such as power of the DC

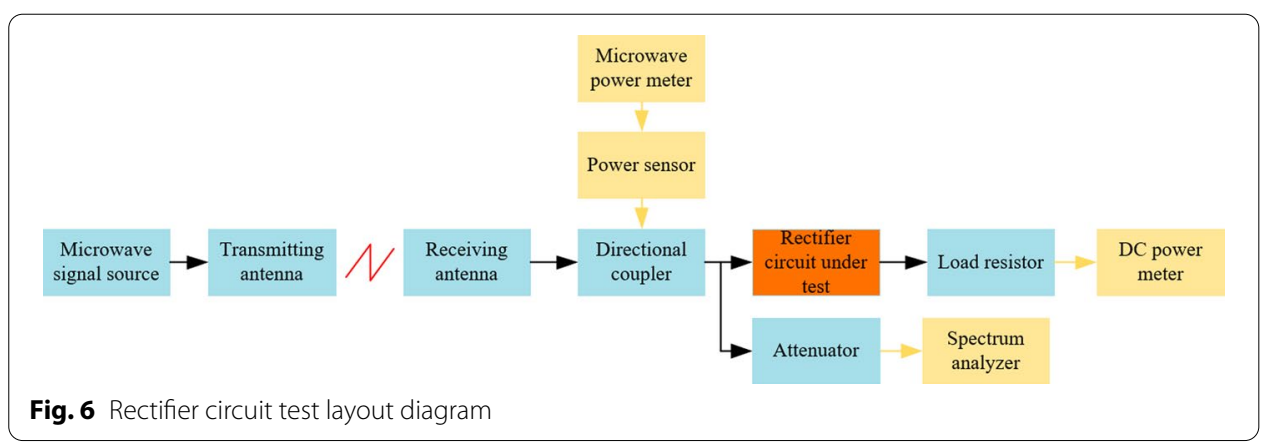



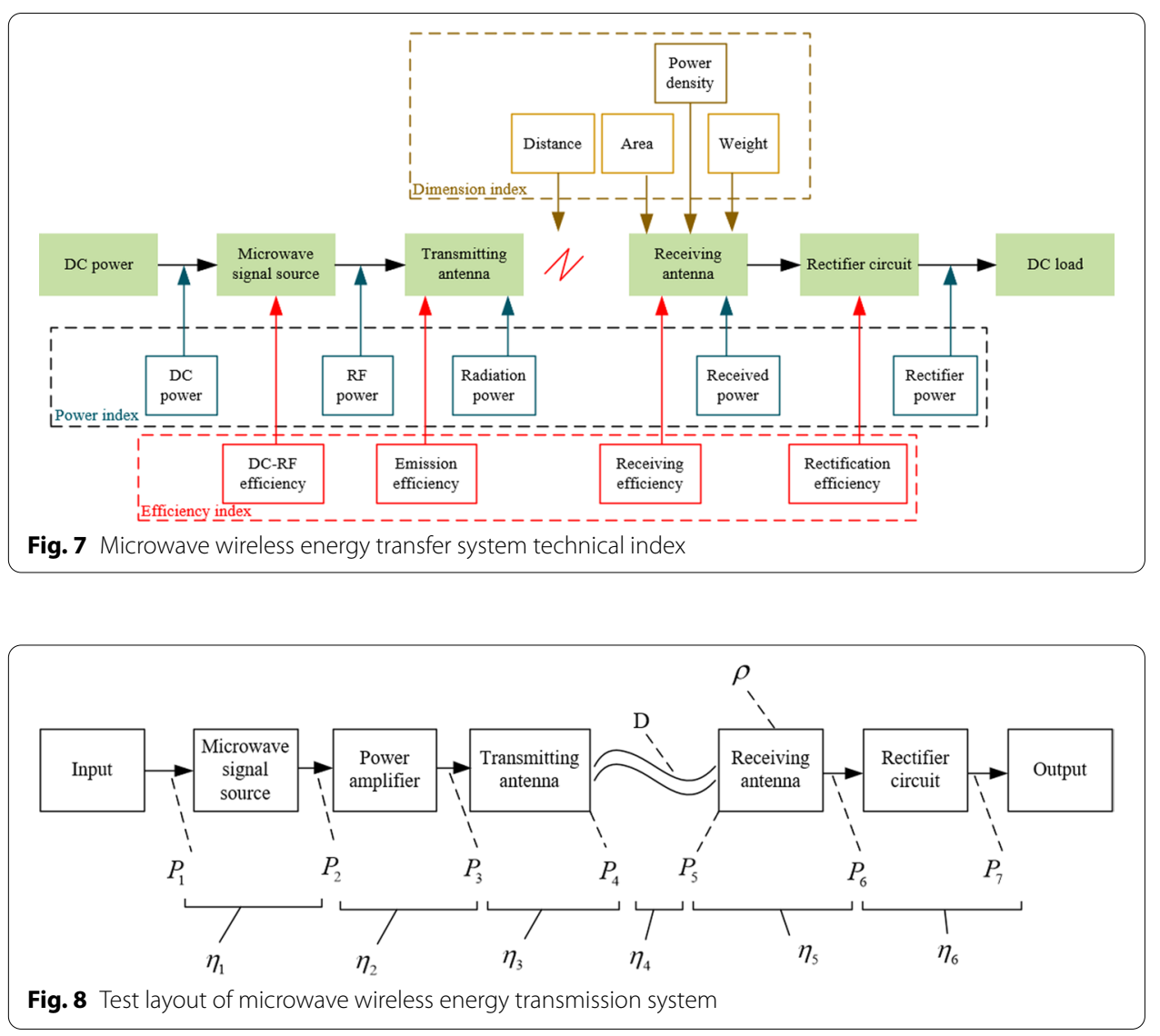

power supply, RF power output from the microwave signal source, power radiated to free space by the transmitting antenna, microwave power received from free space by the receiving antenna, and rectifier circuit output power. Efficiency class indicators include the efficiencies of key system components, such as the efficiency of the microwave signal source to convert the DC signal into an RF signal, efficiency of the transmitting antenna, efficiency of the receiving antenna, and the rectification efficiency. Size class indices are mainly used to evaluate the transmission distance, lightness of the receiving antenna, etc., where the lightness can be evaluated from the area power density and weight power density.

\subsubsection{Test and evaluation methods}

A microwave wireless energy transfer system was built, and the test points were laid out as in Fig. 8.

The metrics in Fig. 8 are defined as shown in Table 1.

According to the connection test diagram shown in Fig. 8, given the input DC power $P_{1}$ and the measured power $P_{7}$ after rectification by the rectifier circuit, the overall efficiency of the microwave wireless energy transmission system can be expressed as $\eta=\frac{P_{7}}{P_{1}}$.

While measuring the system efficiency, the input and output power and efficiency of the main components of the system are also given. The purpose is to analyze the system energy flow further and visually demonstrate the loss in energy transmission, which 
Table 1 Indicator definitions

\begin{tabular}{ll}
\hline$P_{1}$ & Input power of microwave signal source \\
$P_{2}$ & Output power of microwave signal source (input power of power amplifier) \\
$P_{3}$ & Output power of power amplifier (input power of transmitting antenna) \\
$P_{4}$ & Output power of transmitting antenna \\
$P_{5}$ & Input power of receiving antenna \\
$P_{6}$ & Output power of receiving antenna (input power of rectifier circuit) \\
$P_{7}$ & Output power of rectifier antenna \\
$\eta_{1}$ & Conversion efficiency of microwave signal source \\
$\eta_{2}$ & Power amplifier conversion efficiency \\
$\eta_{3}$ & Transmitting antenna conversion efficiency \\
$\eta_{4}$ & Spatial transmission efficiency \\
$\eta_{5}$ & Conversion efficiency of receiving antenna \\
$\eta_{6}$ & Conversion efficiency of rectifier circuit \\
$D$ & Transmission distance \\
$\rho$ & Power density, including area power density and weight power density \\
\hline
\end{tabular}

facilitates the analysis of factors affecting the system efficiency and provides guidance for improving the system efficiency. The tests include changing the DC power input, the distance of the transceiver antenna, and the parameters of a particular component of the system while keeping the rest of the components unchanged, and thus enable each of these effects to be analyzed individually.

\section{Discussion}

Microwave wireless power transmission is one of the important ways of long-distance energy transmission and has broad application prospects. Through consulting a large number of literature, it is known that although the technology is relatively mature, a perfect evaluation index system has not been formed, which is extremely unfavorable to its application and development. At present, the evaluation index of microwave wireless power transmission focuses on the transmission efficiency of the system, and pays less attention to the performance of components and applications. In fact, the performance of components directly affects the performance of the system, and the actual application performance of the system is often related to many factors, which is far from reaching the ideal state.

Microwave wireless power transmission system is mainly composed of signal generator, power amplifier, transceiver antenna, rectifier circuit and other key components. This paper adopts the idea of splitting and reorganization, analyzes the functions of each component in the microwave wireless power transmission system, puts forward the indicators that may affect the transmission efficiency of the system, and then puts the components as the tested object into the microwave wireless power transmission system for testing, so as to form a perfect microwave wireless power transmission technology index system and test and evaluation method. It can not only evaluate the performance of a single component, but also evaluate the impact of components on the system performance and the actual application performance of the system, so as to provide a reference basis for the development of microwave wireless power transmission technology. 


\section{Conclusion}

To promote the development of microwave wireless energy transfer system technology, extensive research has been conducted on the technical indices and test methods for microwave wireless energy transfer systems, including analyzing the topology of microwave wireless energy transfer systems, establishing the technical indices of key components and system performance, and constructing corresponding test and evaluation methods. Although many important advances have been made, there are still some aspects that require further exploration.

(1) Signal generator, power amplifier, transceiver antenna, and other technologies should be studied to improve the conversion efficiency of the core components further and strengthen the miniaturization, lightness, assignability, electromagnetic compatibility, and other types of performance of microwave wireless energy transmission systems.

(2) To assess effectiveness, the evaluation index system should be optimized further, forming a basis for testing the entire chain of microwave wireless energy transmission systems. The development of standards for the interface, transmission frequency, communication protocol, interoperability, energy management, etc. should be coordinated.

(3) Because of the complexity of microwave wireless energy transmission system testing and evaluation, it is necessary to improve the testing instrumentation further, determine the corresponding test specifications, and develop a reasonable evaluation method to provide a powerful technical means for the development of microwave wireless energy transmission systems.

\section{Acknowledgements}

We are grateful to the anonymous reviewers who have contributed to the enhancement of the paper's completeness with their valuable suggestions.

\section{Authors' contributions}

This paper is completed with the cooperation of five authors. CFW proposed the research direction. WLX proposed the research methods. CLZ analyzed the data and wrote this manuscript. MYW carried out the translation work. XDW proofread the paper. All authors read and approved the final manuscript.

Funding

Not applicable.

Availability of data and materials

The datasets used and/or analyzed during the current study are available from the corresponding author on reasonable request.

\section{Declarations}

Ethics approval and consent to participate

Not applicable.

Consent for publication

We agree to the publication of the paper

Competing interests

The authors declare that they have no competing interests.

Author details

${ }^{1}$ Institute of System Engineering, Academy of Military Science, Beijing, China. ${ }^{2}$ Beijing Representative Office, Beijing, China. 
Published online: 02 March 2022

\section{References}

1. H. Ma, D. Shi, Microwave wireless power transmission technology: application prospects and development suggestions in aerospace. Space Electron. Technol. 9, 1-5 (2012)

2. M. Alotaibi, Low noise moving target detection in high resolution radar using binary codes. EURASIP J. Adv. Signal Process. 8, 1-19(2021)

3. C.R. Jaffe, I. Bernd, Microwave and millimeter wave power beaming. IEEE J. Microwaves 1, 229-259 (2021)

4. K.S. Kim, J. Kim, Preliminary operational aspects of microwave-powered airship drone. Int. J. Micro Air Veh. 11 $175682931986136(2019)$

5. S.M. Maekawa, S. Nakamura, The plan of microwave power transmission development for SSPS and its industry application, in 2018 Asia-Pacific Microwave Conference (APMC) (2018)

6. X. Liu, X. Zhang, Noma-based resource allocation for cluster-based cognitive industrial internet of things. IEEE Trans. Ind. Inform. 16(8), 5379-5388 (2020)

7. W.L. Xin Liu, Zhai Xiangping, C. Wu, QoS-guarantee resource allocation for multibeam satellite industrial internet of things with NOMA. IEEE Trans. Ind. Inform. 17(3), 2052-2061 (2021)

8. X. Liu, X. Zhang, Rate and energy efficiency improvements for 5G-based loT with simultaneous transfer. IEEE Internet Things J. 6(4), 5971-5980 (2019)

9. X.Z. Xin Liu, M. Jia, 5G-based green broadband communication system design with simultaneous wireless information and power transfer. Phys. Commun. 28, 130-137 (2018)

10. H.M.X.X. Li, A highly efficient microwave wireless power transmission system. Space Electron. Technol. 1, 1-5 (2016)

11. A.R. Farr, L. Foged, IEEE Standard for Definitions of Terms for Antennas (IEEE, Piscataway, 2014)

12. B. Xu, Research on techology of microwave wireless charging circuit, Master's thesis, School of Electronic Science and Engineering, 2019

13. X. Luo, Research on nonlinear devices in microwave wireless power transmission system, Master's thesis, University of Electronic Science and Technology of China, 2020

14. P. Wang, W. Zhu, Modern Microwave Engineering Survey (Publishing House of Electronics Industry, Beijing, 2014)

15. W. Wang, Research of reconfigurable mems microwave antennas, Master's thesis, School of Electronic Science and Engineering Southeast University, 2014

16. Y. Li, Research on wireless power supply system of mobile object based on microwave, Master's thesis, North China University of Science and Technology, 2017

17. X. Pan, L. Yang, Downlink multi-user algorithms for millimeter-wave wideband linear arrays on PD-NOMA-based squint steering beams. EURASIP J. Adv. Signal Process. 60, 1-26 (2021)

18. N. Shinohara, N. Hayashi, S. Kojima, New beam forming technology for narrow beam microwave power transfer, in The 8th Asia-Pacific Conference on Antennas and Propagation (APCAP 2019): 4-7 August 2019, Incheon (2019)

19. J. Liu, Research on high-efficiency rectifying circuit and antenna for microwave power transmission, PhD thesis, South China University of Technology, 2018

20. Y.D. Dong, Y. Wang, Focused microwave power transmission system with high-efficiency rectifying surface. IET Microw. Antenna Propag. 12(5), 808-813 (2018)

21. F. Li, X.L.J.W.K.Z.K. Lam, L. Wang, Joint pricing and power allocation for multibeam satellite systems with dynamic game model. IEEE Trans. Veh. Technol. 67(3), 2398-2408 (2018)

\section{Publisher's Note}

Springer Nature remains neutral with regard to jurisdictional claims in published maps and institutional affiliations.

\section{Submit your manuscript to a SpringerOpen ${ }^{\circ}$ journal and benefit from:}

- Convenient online submission

Rigorous peer review

- Open access: articles freely available online

- High visibility within the field

- Retaining the copyright to your article

Submit your next manuscript at $\gg$ springeropen.com 